\title{
Reparación de tendones flexores con WALANT: técnica quirúrgica y lesiones asociadas
}

\author{
Flexor tendon repair with WALANT technique: \\ surgical technique and concomitant lesions
}

\author{
Ranulfo Romo Rodríguez, ${ }^{\star}$ Roberto Pablo Pareyón Valeroł
}

\section{RESUMEN}

Introducción: La anestesia local sin torniquete y con el paciente despierto (WALANT, por sus siglas en inglés) ha ganado popularidad en las últimas décadas para realizar diversas cirugías de la mano y muñeca. Consideramos que esta técnica tiene un gran valor particularmente en la cirugía de tendones flexores debido a que permite corroborar de forma dinámica la resistencia de la reparación y el recorrido tendinoso adecuado. Además involucra de forma activa al paciente durante el acto quirúrgico permitiéndole constatar la calidad y funcionalidad de la reparación motivándolo así para realizar el proceso de rehabilitación. Objetivo: Demostrar, mediante una serie de pacientes, la capacidad de evitar complicaciones y obtener buenos resultados con lesiones de tendones flexores y uso de técnica WALANT. Material y métodos: En este trabajo se presenta una serie de casos de ocho pacientes operados de cirugía de tendones flexores en los que se utilizó la técnica anestésica tipo WALANT. Estos casos ejemplifican la totalidad del espectro de la cirugía de tendones flexores incluyendo la reparación primaria, la reconstrucción en uno y dos tiempos y la tenólisis. Resultados: Los resultados logrados con esta técnica quirúrgica son equiparables a los logrados con las técnicas tradicionales, pero con las ventajas antes mencionadas y a un menor costo. No se presentaron complicaciones atribuibles a la técnica anestésica. Conclusión: Con esta serie de pacientes se concluye que la técnica WALANT es altamente recomendada para la cirugía de reconstrucción de tendones flexores, por su adecuada verificación transoperatoria y la baja tasa de complicaciones.

Palabras clave: WALANT, tendones, tenólisis, isquemia.

Nivel de evidencia: IV

\begin{abstract}
Introduction: Wide awake local anesthesia no tourniquete has gained popularity in recent decades to perform various hand and wrist surgeries. We consider that this technique has a great value, particularly in flexor tendon surgery because it allows to dynamically corroborate the resistance of the repair and the adequate tendon path. It also actively involves the patient during the surgical act, allowing him to verify the quality and functionality of the repair, thus motivating him to do the rehabilitation process. Objective: Is to demonstrate, through a series of patients, tha bility to avoid complications and obtain good results with WALANT technique and reconstruction of flexor tendon injuries. Material and methods: This paper presents a series of eight patients undergoing surgery of flexor tendons in wich the WALANT technique was used. Thes cases exemplify the entire spectrum of flexor tendon surgery including primary repair, reconstruction in one and two times and tenolysis. Results: The results achieved with this surgical technique are comparable to those achieved with traditional techniques but with several advantages such as lower cost, less complications and the capacity to evaluate the repair during surgery. There were no complications attributable to the anesthesic technique. Conclusion: Wiht this series of patients it is concluded that de WALANT technique is highly recommended for flexor tendon reconstruction surgery, due to its adequate transoperative verification and the low complication rate and low cost.
\end{abstract}

Keywords: WALANT, tendon, tenolysis, ischemia.

Level of evidence: IV

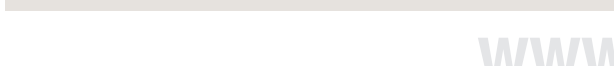 \\ * Cirujano de extremidad superior y microcirugía. \\ ‡ Residente de segundo año de Traumatología y Ortopedia.}

Centro Médico ABC.

Correspondencia: Dr. Ranulfo Romo Rodríguez Calle Sur 132 Núm. 122, Col. Las Américas, 01120,

Álvaro Obregón, Ciudad de México. Tel: 5535555544 E-mail: ropareyon@gmail.com

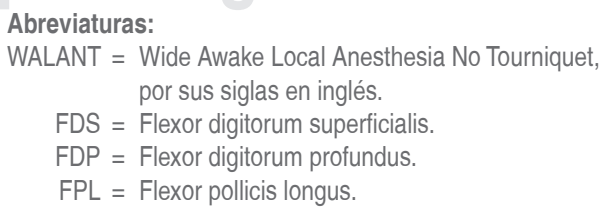

Recibido para publicación: 18/01/2020. Aceptado: 02/03/2020. 


\section{INTRODUCCIÓN}

Desde que Sterling Bunnel sentó las bases de la cirugía de mano mencionando que operar una mano sangrante equivale a reparar un reloj dentro de un tintero, en su mayoría ésta se ha realizado con torniquete para crear un campo sin sangre y mejorar la visualización. La isquemia prolongada de la extremidad superior genera un descenso de $\mathrm{pH}$ que sumado a la compresión mecánica a nivel del torniquete provoca dolor intenso al paciente, lo que genera la necesidad de realizar los procedimientos bajo anestesia general, bajo sedación profunda o con bloqueos de plexo braquial.

Tradicionalmente se ha contraindicado el uso de epinefrina en partes acras debido al temor a que ésta provoque necrosis secundaria a la vasoconstricción. Sin embargo, en la actualidad, existe amplia evidencia acerca de la seguridad de utilizar epinefrina en la mano incluyendo los dedos. Esto ha permitido generar la técnica WALANT (Wide Awake Local Anesthesia No Tourniquet) que puede ser utilizada para casi todas las cirugías realizadas en la mano y muñeca. La evidencia ha probado que la vasoconstricción es segura en la mano, incluyendo los dedos, lo que ha eliminado la necesidad de un torniquete en la mayoría de las cirugías de mano, ya que la vasoconstricción generada es mundialmente aceptada. ${ }^{1-6}$

La cirugía de tendones flexores es una de las más demandantes y exigentes dentro de la cirugía de mano. Requiere de reparaciones resistentes que permitan el movimiento inmediato para evitar adherencias. Además, estas reparaciones deben de tener un volumen adecuado para permitir al tendón deslizarse dentro del sistema de poleas para permitir un recorrido tendinoso completo. Debido a esto, opinamos que la técnica WALANT es particularmente valiosa en la cirugía de tendones flexores, ya que se puede probar la calidad de la reparación tendinosa mediante el movimiento activo del paciente. Esto permite detectar de forma primaria casos en los que la reparación no es suficientemente fuerte para resistir esfuerzos flexores fisiológicos o casos en los que el volumen de la reparación es tal que no permite el deslizamiento a través del sistema de poleas. Al detectar estas complicaciones se pueden tomar medidas como son repetir la tenorrafia y resecar o ventilar poleas para permitir el deslizamiento adecuado del tendón.7,8

El objetivo de este estudio es reportar nuestra experiencia en cirugía de tendones flexores de la mano utilizando la técnica WALANT.

\section{MATERIAL Y MÉTODOS}

Entre septiembre de 2013 y noviembre de 2016 se realizaron 11 cirugías en ocho pacientes con diversas lesiones de tendones flexores. Todos los procedimientos fueron realizados por un solo cirujano.

Los pacientes eran seis hombres y dos mujeres entre las edades de 12 y 47 años. La serie incluye todos los procedimientos básicos de la cirugía de tendones flexores (Tabla 1), incluyendo la reparación de un flexor único, reparación de FDS y FDP en un solo dedo, reparación de flexores en múltiples dedos, reconstrucción de FDP en un tiempo, reconstrucción de FDP en dos tiempos, reparación de FPL y tenólisis.

Adicionalmente, en tres pacientes se realizó cirugía de nervios digitales, estos procedimientos fueron: una neurólisis, una neurorrafia primaria y una reconstrucción con injerto de nervio sural.

Se contó con el registro fotográfico y en video de los ocho pacientes así como el seguimiento de la evo-

Tabla 1: Pacientes por grupo de edad y sexo.

\begin{tabular}{|c|c|c|c|c|c|c|c|}
\hline Paciente & $\begin{array}{l}\text { Edad } \\
\text { (años) }\end{array}$ & Sexo & Dedo & Sangrado (mL) & $\begin{array}{c}\text { Tiempo } \\
\text { quirúrgico (min) }\end{array}$ & Complemento & Tenólisis \\
\hline 1 & 46 & $\mathrm{~F}$ & Índice & 10 & 60 & No & No \\
\hline 2 & 47 & $\mathrm{~F}$ & $3^{\circ}$ y $4^{0}$ & 30 & 195 & Nervio cubital & Sí \\
\hline 3 & 25 & M & Dedo medio & 5 & 150 & Cierre herida & No \\
\hline 4 & 32 & M & Pulgar & 5 & 250 & Nervio radial y cubital & No \\
\hline 5 & 37 & M & Pulgar & 5 & 105 & No & No \\
\hline 6 & 39 & M & Índice & 10 & 180 & $\begin{array}{l}\text { Reconstrucción en dos } \\
\text { tiempos + poleas A3 y A5 }\end{array}$ & No \\
\hline 7 & 26 & M & Índice & ---- & $-\ldots$ & & \\
\hline 8 & 12 & M & Meñique & ---- & ---- & & \\
\hline
\end{tabular}




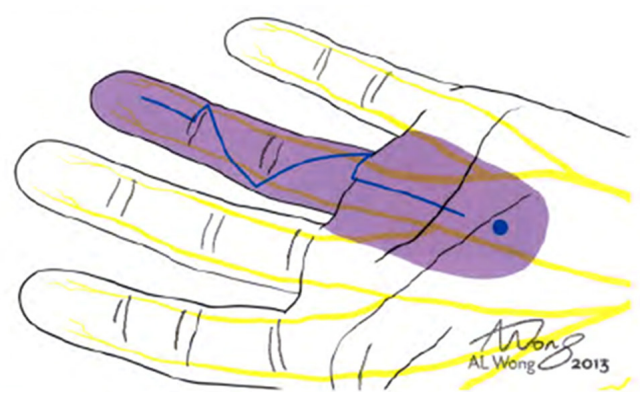

Figura 1: Se infiltra con $15 \mathrm{~mL}$. Se comienza con $10 \mathrm{~mL}$ a $1 \mathrm{~cm}$ de donde comienza la incisión en región proximal.

lución clínica en el consultorio. Se recabaron los expedientes clínicos y las notas quirúrgicas en los casos posibles. De lo anterior se registraron el tiempo quirúrgico, el sangrado, las complicaciones, la necesidad de procedimientos secundarios y el resultado clínico.

\section{Técnica quirúrgica}

Todas las cirugías se realizaron en quirófano (ninguna en consultorio ni en urgencias). Se realizó una asepsia inicial del sitio quirúrgico posterior, a lo cual se infiltró con lidocaína y epinefrina $20 \mathrm{mg} / 0.005$ $\mathrm{mg} / 1 \mathrm{~mL}$ (Pisacaína, Pisa) hasta que la zona quirúrgica se volviera turgente con el anestésico. Posterior a esto se realizó el protocolo quirúrgico habitual (asepsia de toda la extremidad, colocación de campos, etcétera) para permitir que durante este tiempo la epinefrina generara el efecto vasoconstrictor.

Se abordaron los tendones mediante incisiones volares de Brunner y/o prolongando las heridas traumáticas en caso de existir. En los casos de las tenorrafias término-terminales, todas fueron realizadas con Supramid $^{\circledR}$ 4-0 excepto un caso en el que fue utilizado Nylon 4-0. En todos los casos se logró un mínimo de seis pases de sutura central a nivel de la reparación y se reforzó con una sutura epitendinosa continua de Nylon 6-0. En los casos en los que se requirió de un anclaje óseo en la falange distal, éste fue realizado con técnica de "pull-out» saliendo en la placa ungueal en dos ocasiones y en una ocasión con anclas de 1.3 mm (Mytek). En los casos que requirieron de injerto tendinoso para la reconstrucción se utilizó en dos ocasiones el palmaris longus y en otro un extensor de los dedos del pie. En el caso que fue tratado mediante técnica de Hunter para reconstrucción en dos tiempos se utilizó un espaciador de silicona de calibre 3 (Wright Medical). ${ }^{9,10}$

Las neurorrafias fueron realizadas con técnicas microquirúrgicas con Nylon 9-0 y el injerto nervioso requerido en un caso fue de nervio sural ipsilateral, en el que también se aplicó la técnica WALANT. Los tendones retraídos en la palma fueron recabados con la técnica de Sourmelis-McGrouther. ${ }^{8}$ En todos los casos se respetó un mínimo de $75 \%$ de la polea A2, mientras que las demás poleas, incluyendo A4, fueron liberadas según la necesidad para permitir el recorrido tendinoso. Posterior a la reparación tendinosa se pidió al paciente realizar los movimientos de flexión y extensión completos para probar la resistencia de la reparación así como el recorrido tendinoso. En caso de ocurrir alguna falla se realizaron las medidas pertinentes. Adicionalmente, los casos fueron documentados mediante fotografías y videos transquirúrgicos que fueron compartidos con el paciente.

Posterior a la cirugía, todos los pacientes fueron inmovilizados con una férula dorsal con la muñeca en neutro, flexión a $70^{\circ}$ de las articulaciones metacarpofalángicas e interfalángicas a $0^{\circ}$.

Todos los pacientes fueron rehabilitados mediante el protocolo de Duran modificado (Figuras 1 a 5). ${ }^{11}$

\section{RESULTADOS}

De los ocho pacientes sometidos a 11 procedimientos, se pudieron recabar los expedientes hospitalarios y las notas quirúrgicas de seis pacientes sometidos a ocho procedimientos. El tiempo quirúrgico en los procedimientos de estos seis pacientes fue varia-

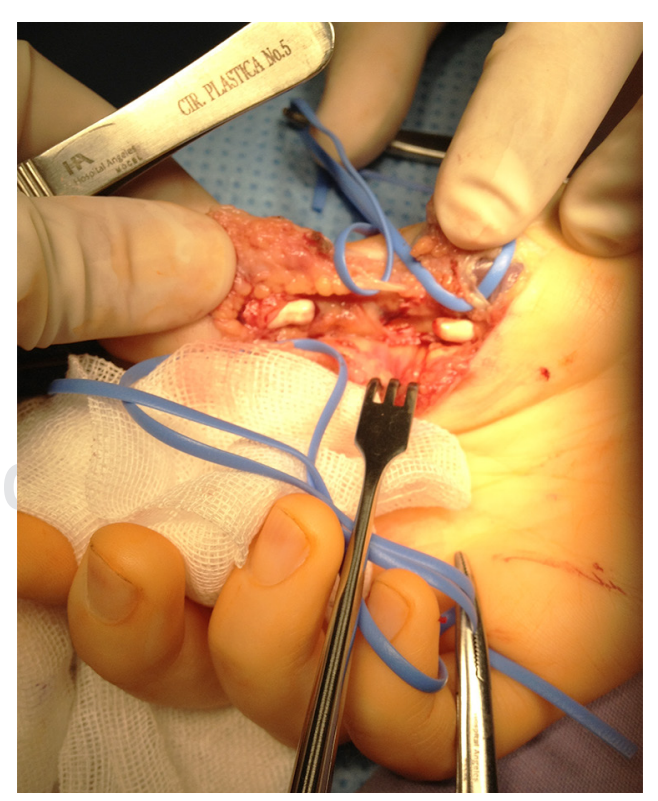

Figura 2: Localización de ambos cabos, tanto proximal como distal separados con cordones azules. 


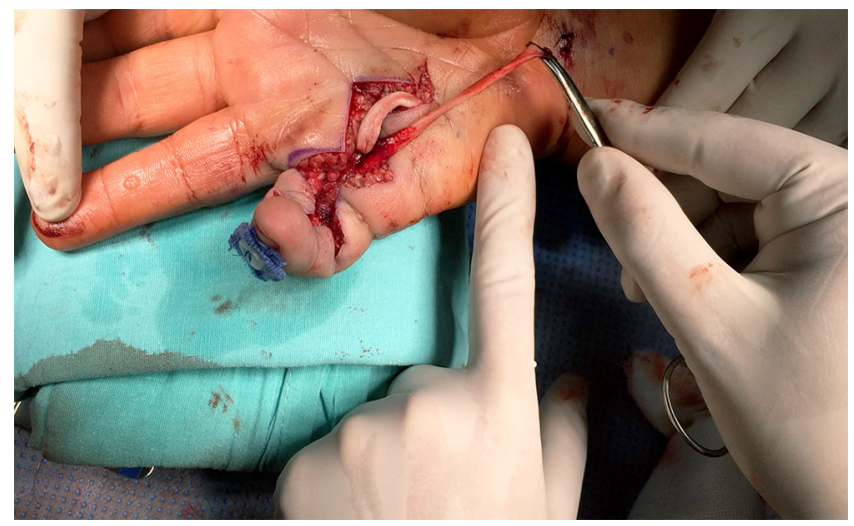

Figura 3: Se realiza medición del cabo proximal al cabo distal para su posterior reparación con adecuada tensión.

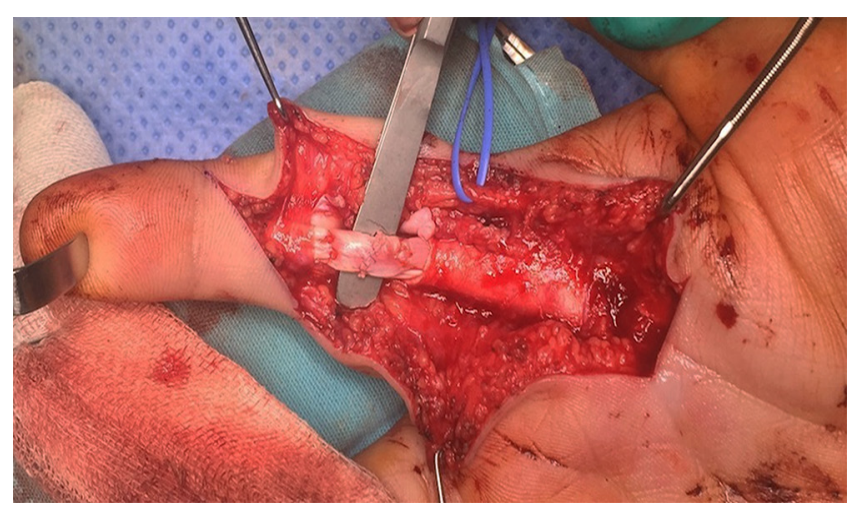

Figura 4: Reparación final usando lo descrito en la técnica quirúrgica.

ble dependiendo de la necesidad de procedimientos complementarios, siendo el más corto de una hora y el más largo de cinco horas, teniendo un promedio en los seis pacientes de dos horas y 45 minutos.

En cuestión de sangrado el paciente con mayor cantidad fue de $15 \mathrm{~mL}$ y el de menor cantidad fue de 5 $\mathrm{mL}$, con promedio de $10 \mathrm{~mL}$ en los 10 procedimientos.

Se tiene un total de ocho pacientes y un total de 11 procedimientos, todos realizados con técnica WALANT. Los resultados están reportados en la Tabla 1 y están descritos en la Tabla 2 . Se puede ver que de los ocho pacientes tenemos involucradas cinco manos derechas y tres izquierdas. Los pacientes son seis masculinos y dos femeninos con promedio de edad de 33 años. Con un promedio de tiempo quirúrgico de 120 minutos por procedimiento. De los pacientes reportados tres fueron dedos índice, dos pulgares, uno medio, uno meñique y uno con dedos medio y anular.

Se realizaron adicionalmente una neurorrafia primaria (paciente operada de anular y medio), una reconstrucción de nervio digital con injerto sural (paciente operado de FPL) y una neurólisis (paciente de operado de técnica de Hunter).

En cinco pacientes obtuvimos resultados excelentes, un paciente obtuvo buenos resultados (reconstrucción de FDP de meñique en un tiempo quirúrgico) y dos resultado malos (reparación de FPL con reconstrucción de nervio y reconstrucción de FDP con técnica de Hunter). Como complicaciones dos pacientes presentaron adherencias, uno de los cuales fue operada nuevamente a los cuatro meses de la cirugía inicial logrando resultados favorables, cabe mencionar que esta paciente fue operada de los FDP del anular y del medio además de ser sometida a una neurorrafia en el dedo medio. El otro paciente que presentó adherencias fue operado de un FPL cuatro semanas después de la lesión inicial y es el que fue sometido a la reconstrucción nerviosa con nervio sural. Este paciente no acudió a rehabilitación como le fue indicado y no quiso someterse a la tenólisis.

Adicionalmente existió un falla de material en el paciente sometido a la técnica de Hunter en quien el injerto de palmaris longus (segundo tiempo quirúrgico) fue anclado a la falange distal del dedo índice con dos anclas de $1.3 \mathrm{~mm}$. Al ser operado en una tercera ocasión, se observó que la falla ocurrió en la interfase ancla-hueso. En el tercer tiempo quirúrgico, le fue colocado un injerto de extensor de los dedos del pie. Este paciente no logró recuperar una flexión activa funcional del dedo.

Se reportan los resultados con la clasificación de Strickland en la Tabla 3.

En la serie tuvimos dos malos resultados antes mencionados: FPL por adherencias (adherencias) y reconstrucción de Hunter (falla de material y adherencias). Al probar de manera activa la calidad de la reparación tendinosa pidiéndole al paciente realizar

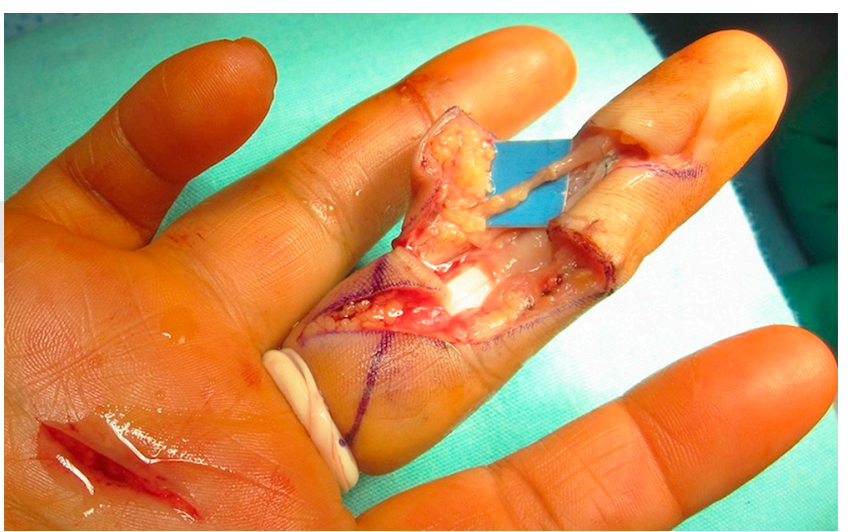

Figura 5: Ejemplo de paciente con necesidad de generar reparación de nervio cubital interdigital, además de la reparación del tendón flexor. 
Tabla 2: Total de procedimientos.

\begin{tabular}{|c|c|c|}
\hline Paciente & Procedimiento 1 & Procedimiento 2 \\
\hline 1 & $\begin{array}{l}\text { Reparación término-terminal } \\
\text { del tendón flexor profundo } \\
\text { índice derecho }\end{array}$ & No \\
\hline 2 & $\begin{array}{l}\text { Reparación término-terminal } \\
\text { tendón flexor dedo medio y } \\
\text { anular + reparación de ner- } \\
\text { vio cubital interdigital dedo } \\
\text { medio mano derecha }\end{array}$ & $\begin{array}{l}\text { Tenólisis de } \\
\text { tendones flexores } \\
\text { de dedo medio y } \\
\text { anular de mano } \\
\text { derecha }\end{array}$ \\
\hline 3 & $\begin{array}{l}\text { Reparación término-terminal } \\
\text { del tendón flexor profun- } \\
\text { do de dedo medio mano } \\
\text { izquierda }\end{array}$ & No \\
\hline 4 & $\begin{array}{l}\text { Reparación término-terminal } \\
\text { del tendón flexor largo del } \\
\text { pulgar derecho + reparación } \\
\text { de nervio radial y cubital in- } \\
\text { terdigital de pulgar derecho }\end{array}$ & No \\
\hline 5 & $\begin{array}{l}\text { Reparación término-terminal } \\
\text { de tendón flexor largo del } \\
\text { pulgar mano izquierda }\end{array}$ & No \\
\hline 6 & $\begin{array}{l}\text { Primer tiempo: reconstruc- } \\
\text { ción de tendón flexor profun- } \\
\text { do de índice derecho + } \\
\text { espaciador + reconstrucción } \\
\text { de poleas A3 y A5 }\end{array}$ & $\begin{array}{l}\text { Segundo tiempo: } \\
\text { toma y aplicación } \\
\text { de injerto de } \\
\text { palmar menor } \\
\text { para reparación } \\
\text { del tendón flexor } \\
\text { profundo del dedo } \\
\text { índice derecho }\end{array}$ \\
\hline
\end{tabular}

flexión activa durante la cirugía, no tuvimos ningún caso de rotura de la reparación, pero sí observamos dos casos en los que los nudos centrales de la neurorrafia se atoraban en bordes de las poleas cruciformes. En estos dos casos se repitieron las tenorrafias situando en otro lugar los nudos para evitar la obstrucción.

Consideramos que ninguna de las complicaciones fue atribuible a la técnica WALANT como técnica de elección para la anestesia.

\section{DISCUSIÓN}

Las lesiones de tendones flexores son lesiones traumáticas que tienen una profunda repercusión en la función de la mano. El tratamiento de estas lesiones ha sido un reto para los cirujanos de mano. Hipócrates ya reconocía la gravedad funcional de la lesión; sin embargo, no distinguía específicamente a los tendones, mismos que llamaba «neurona». Galeno fue el primero en reconocer la diferencia histológica entre tendones y nervios aunque sólo de manera parcial, ya que no re- comendaba manipular o suturar los tendones, ya que ello llevaría a convulsiones, concepto que fue refutado por Meekren en 1682. En el siglo XVIII, Hunter realizó las primeras tenorrafias registradas de la medicina occidental. En 1920, Saloman y Hueck notaron una pobre cicatrización de los tendones flexores, dentro y fuera de su vaina sinovial. Durante la primera mitad del siglo XX, los resultados de las tenorrafias eran pobres, particularmente en la «tierra de nadie» donde el tratamiento de elección era la resección tendinosa y la colocación de injerto para reconstrucción. El punto de inflexión en el tratamiento de las lesiones de tendones flexores no solamente a nivel de la «tierra de nadie» fue en 1967 cuando Kleinert ${ }^{11}$ presentó su técnica y resultados. Desde entonces, se ha reconocido la capacidad del tendón para cicatrizar en forma intrínseca. Se han diseñado múltiples técnicas de reparación, todas enfocadas en ofrecer una resistencia adecuada para un movimiento temprano. Se ha puesto énfasis en el movimiento y rehabilitación temprana para evitar adherencias. Los puntos anteriores han logrado mejorar radicalmente los resultados de la cirugía de tendones flexores. En una de las más grandes series reportadas, Jin Bo Tang ${ }^{12}$ tiene una recolección de 300 pacientes, todos operados por cirujanos de mano con experiencia en tres diferentes hospitales y utilizando el mismo protocolo. Sólo tiene un caso ruptura posterior a la reparación y logró resultados excelentes en rangos de movilidad en un $80 \%$. Lo esencial de este protocolo fue:

1. Una separación de entre $0.7 \mathrm{~mm}$ y $1 \mathrm{~cm}$ entre ambos cabos.

2. Contacto y abultamiento de la reparación entre 10 y $30 \%$ del espesor total del tendón.

3. Utilizar un total de seis pases de suturas a través del núcleo del tendón.

4. Realizar la apertura de la mitad de la polea A2 o incluso toda la polea de A4 sin abrirla más de 2 centímetros.

Tabla 3: Resultados según clasificación Strickland.

\begin{tabular}{cc}
\hline Paciente & Resultados según Strickland $(\%)$ \\
\hline 1 & Excelente $(>75)$ \\
2 & Bueno $(<75 \mathrm{y}>50)$ \\
3 & Excelente $(>75)$ \\
4 & Excelente $(>75)$ \\
5 & Excelente $(>75)$ \\
6 & Regular $(<50 \mathrm{y}>25)$ \\
7 & Excelente $(>75)$ \\
8 & Excelente $(>75)$ \\
\hline
\end{tabular}


5. Realizar movimientos activos posterior a la reparación de total flexión y extensión durante la cirugía para comprobar arcos de movilidad y paso a través de las poleas restantes.

6. En el postoperatorio inmediato se puede realizar activamente la mitad de un puño en varias ocasiones sin uso de ligas ni la necesidad de completar el puño y mantenerlo, un protocolo de rehabilitación muy parecido al de Duran modificado, el cual fue utilizado en el postoperatorio para estos pacientes.

7. Utilizar todo el tiempo necesario durante la cirugía para educar al paciente, ya que se tiene al paciente completamente despierto se pueden dar indicaciones claras de cómo evitar las rupturas tendinosas.

Para cumplir los conceptos de resistencia y movimiento es necesario realizar pruebas transquirúrgicas para constatar la calidad de la reparación. Foucher propone realizar incisiones en el antebrazo distal para traicionar los tendones reparados y así constatar la calidad de la reparación; empero, esto agrega morbilidad el paciente. Otros proponen hacer pruebas de tenodesis con flexo-extensión de la muñeca o comprimir las masas musculares del antebrazo para evaluar al tendón reparado; sin embargo, esto no brinda la fuerza de tracción ni el recorrido tendinoso que ocurre en situaciones fisiológicas o durante la rehabilitación. Consideramos que el mejor método para evaluar los parámetros antes mencionados es con el flexo-extensión activa del paciente mismo que es posible con la técnica anestésica de WALANT, ya que el paciente se encuentra despierto y puede cooperar, además de que al no utilizar torniquete los músculos y nervios de la extremidad superior se mantienen funcionales durante todo el procedimiento.

Donald LaLonde acuñó el término WALANT y ha sido el responsable de la difusión amplia de esta técnica desde su publicación en 2005; sin embargo, esta técnica se ha utilizado en Canadá por más de 40 años. Adicionalmente, desde los años 2000, ha surgido evidencia amplia acerca de la seguridad de utilizar epinefrina en la mano. Chowdhry en 2010 y Lalonde en 2005 reportaron series de 1,111 y 3,110 pacientes consecutivos en quien se aplicó epinefrina con lidocaína sin presentar ningún caso de necrosis. ${ }^{8}$

El temor al uso de la epinefrina se remonta a las décadas de los 20 a 40 del siglo pasado cuando la epinefrina era combinada con procaína en vez de lidocaína. La procaína disminuye importantemente el $\mathrm{pH}$ (13.6) de los tejidos, lo cual era responsable de provocar la temida necrosis de los tejidos y no la epinefrina. En la técnica publicada por Lalonde, se infiltra lidocaína con epinefrina al $1 \%$ y se tiene en cuenta una dosis máxima de $7 \mathrm{mg} / \mathrm{kg}$, lo cual permite utilizar de manera segura $49 \mathrm{~mL}$ en un paciente de $70 \mathrm{~kg}$. En México la lidocaína con epinefrina es al 2\% (Pisacaína, Pisa), lo cual nos reduce a la mitad el volumen a infiltrar. No obstante, en ningún caso se llegó a la dosis de $7 \mathrm{mg} /$ $\mathrm{kg}$. Además, se menciona que el efecto anestésico y vasoconstrictor se mantiene diluyendo la pisacaína con una relación de $1 / 4$ con la posibilidad de utilizar cuatro veces más volumen al habitual, lo cual no ha sido necesario. Se menciona que en el caso remoto de que la infiltración provocara una isquemia severa o prolongada, la fentolamina puede revertir el efecto vasoconstrictor de la epinefrina; sin embargo, no se cuenta con este medicamento en México. En nuestra serie de casos, ninguno presentó isquemia severa o prolongada. Es importante mencionar que la técnica WALANT no provee un lecho totalmente exangüe como el obtenido con torniquete, pero sí permite la visualización de todas las estructuras necesarias realizando una hemostasia cuidadosa. ${ }^{13}$

La necesidad de procedimientos secundarios está reportada entre 5 y $25 \%$ de los casos de la cirugía de tendones flexores. Nosotros tuvimos tres eventos que requirieron una intervención no planeada. El primero se trató de la paciente en quien se repararon los flexores profundos del dedo medio y del anular. A los tres meses de operada el movimiento pasivo era mayor al activo, por lo que a los cuatro meses se realizó una tenólisis. Debido a su situación socioeconómica la paciente no tuvo acceso a fisioterapia en las primeras semanas después de su cirugía inicial. Después de la tenólisis, ya se encontraba recibiendo fisioterapia, por lo que la pudo continuar después del procedimiento secundario, lo cual puede explicar esta evolución clínica. Otro paciente quien fue sometido a reconstrucción de nervio digital en el pulgar y reparación de flexor pollicis longus también presentó adherencias diagnosticadas por tener un mayor movimiento pasivo que activo. ${ }^{14} \mathrm{El}$ paciente no acudió a fisioterapia después de la cirugía a pesar de que le fue indicada, adicionalmente, declinó una segunda intervención. El tercer evento inesperado correspondió a la rotura del anclaje dista del paciente sometido al segundo tiempo de Hunter. Como se mencionó en los resultados, la falla fue en la interacción ancla-hueso. El paciente fue reintervenido para colocar un injerto de extensor de dedo del pie, pero a pesar de recibir fisioterapia no alcanzó $90^{\circ}$ de flexión digital total. Este paciente tenía una lesión crónica ocasionada cuatro años antes al sufrir una herida cortocontundente con un espolón de gallo. Al momento de la cirugía inicial tenía fibrosis 
extensa y daño de la vaina flexora. A pesar de que se hizo la reconstrucción en dos tiempos es posible que la fibrosis y el estado general del dedo aumentaran las exigencias de tracción llevando a la rotura.

Consideramos que ninguna de las complicaciones referidas fueron atribuibles a la técnica anestésica de WALANT. Por el contrario, gracias a la cooperación del paciente al realizar flexo-extensión activa, pudimos corroborar la resistencia de la reparación y el deslizamiento tendinoso durante la cirugía en todos los casos. No hubo ningún caso transquirúrgico de rotura de la reparación, pero sí hubieron dos casos en los que los nudos de la reparación se atoraban en las poleas, lo cual pudo ser corregido gracias a la técnica de WALANT. Además de lo antes mencionado, consideramos que es de sumo valor que el paciente pueda constatar la calidad de la reparación tendinosa durante la cirugía y posteriormente a ella para que se motive a realizar en forma adecuada la rehabilitación.

Aproximadamente 20\% de todos los cirujanos de mano en Estados Unidos utilizan la técnica de WALANT para realizar reparaciones tendinosas a pesar de su «reciente» descubrimiento. ${ }^{15}$ Lo que hace esta técnica realmente especial es la capacidad de poder generar flexión y extensión total durante la cirugía de un tendón recientemente reparado. Incluso si el tendón tiene una separación de $>1 \mathrm{~cm}$ como pasa en $7 \%$ de los casos o que no hay un $30 \%$ de abultamiento de la reparación, se puede corregir en la misma cirugía, lo que genera un riesgo de ruptura posterior muy bajo. ${ }^{8}$

Si el tendón no pasa tan fácilmente a través de las poleas el cirujano puede cortar hasta $>2 \mathrm{~cm}$ para facilitar este trabajo, incluyendo polea A2 y polea A4 que anteriormente se tenía la idea de no tocar por el riesgo de tener complicación de tendones en cuerda de violín, lo que obviamente también genera una necesidad de tenólisis mucho menor que antes.

\section{CONCLUSIÓN}

La técnica de WALANT se ha incrementado de manera importante en todo el mundo y cada vez son más los procedimientos que se pueden realizar con esta técnica tanto en mano como en muñeca. Consideramos que es de particular beneficio en la cirugía de tendones flexores, ya que podemos corroborar la resistencia de la reparación y el adecuado deslizamiento tendinoso; también, brinda la oportunidad de educar el paciente acerca de su procedimiento. Todo lo anterior tiene el potencial para mejorar resultados en la cirugía de tendones flexores. Adicionalmente, los costos de la cirugía utilizando la técnica anestésica WALANT se pueden ver reducidos sustancialmente. Recomendamos utilizar esta técnica en todos los casos de cirugía de tendones flexores excepto cuando el paciente no sea cooperador, exista alergia a los componentes o exista alguna angiopatía que contraindique el uso de epinefrina.

\section{BIBLIOGRAFÍA}

1. Fitzcharles-Bowe C, Denkler K, Lalonde D. Finger injection with high-dose (1:1,000) epinephrine: Does it cause finger necrosis and should it be treated? Hand (N Y). 2007; 2 (1): 5-11.

2. Nodwell T, Lalonde D. How long does it take phentolamine to reverse adrenaline-induced vasoconstriction in the finger and hand? A prospective, randomized, blinded study: The Dalhousie project experimental phase. Can J Plast Surg. 2003; 11 (4): 187-190.

3. Mckee DE, Lalonde DH, Thoma A, Dickson L. Achieving the optimal epinephrine effect in wide awake hand surgery using local anesthesia without a tourniquet. Hand (N Y). 2015; 10 (4): 613-615.

4. Lalonde DH, Wong A. Dosage of local anesthesia in wide awake hand surgery. J Hand Surg Am. 2013; 38 (10): 2025-2058.

5. Lalonde DH. Wide awake hand surgery. New York: Thieme; 2016.

6. Tang JB. Wide awake hand surgery. Shanghai (China): Shanghai Scientific \& Technical Publishers; 2017.

7. Higgins A, Lalonde DH, Bell M, McKee D, Lalonde JF. Avoiding flexor tendon repair rupture with intraoperative total active movement examination. Plast Reconstr Surg. 2010; 126 (3): 941-945.

8. Lalonde DH. Wide-awake flexor tendon repair. Plast Reconstr Surg. 2009; 123 (2): 623-625.

9. Rhee PC, Fischer MM, Rhee LS, McMillan H, Johnson AE. Cost savings and patient experiences of a clinic-based, wideawake hand surgery program at a military medical center: a critical analysis of the first 100 procedures. J Hand Surg Am. 2017; 42 (3): e139-e147.

10. Chatterjee A, McCarthy JE, Montagne SA, Leong K, Kerrigan CL. A cost, profit, and efficiency analysis of performing carpal tunnel surgery in the operating room versus the clinic setting in the United States. Ann Plast Surg. 2011; 66 (3): 245-248.

11. Cetin A, Dinçer F, Keçik A, Cetin M. Rehabilitation of flexor tendon injuries by use of a combined regimen of modified Kleinert and modified Duran techniques. Am J Phys Med Rehabil. 2001; 80 (10): 721-728.

12. Tang JB, Zhou X, Pan ZJ, Qing J, Gong KT, Chen J. Strong digital flexor tendon repair, extension-flexion test, and early active flexion: experience in 300 tendons. Hand Clin. 2017; 33 (3): 455-463.

13. Lalonde D, Bell M, Benoit P, Sparkes G, Denkler K, Chang P. J A multicenter prospective study of 3,110 consecutive cases of elective epinephrine use in the fingers and hand: the Dalhousie Project clinical phase. Hand Surg Am. 2005; 30 (5): 1061-1067.

14. Lilly SI, Messer TM. Complications after treatment of flexor tendon injuries. J Am Acad Orthop Surg. 2006; 14 (7): 387-396.

15. Gibson PD, Sobol GL, Ahmed IH. Zone II flexor tendon repairs in the united states: trends in current management. $J$ Hand Surg Am. 2017; 42 (2): e99-e108. 